\title{
Therapeutic communities helped people to recover from substance abuse and implement "new lives"
}

Marcus MT. Changing careers: becoming clean and sober in a therapeutic community. Qual Health Res 1998 Jul;8:466-80.

\section{Question}

How do people recover from substance abuse in long term therapeutic communities?

\section{Design}

Grounded theory.

\section{Setting}

3 university sponsored, nurse managed, long term residential clinics in southwestern US. The goals of the clinic programme were to help participants change attitudes; assume responsibility for actions; and work towards congruence of behaviour, feelings, values, and attitudes for a drug free lifestyle. Using behaviour modification, encounter groups, occupational therapy, recovering addicts as cotherapists, and resocialisation, participants took up to 30 months to progress through orientation, treatment, and re-entry.

\section{Patients}

13 adults with substance abuse problems (4 with cocaine, 4 with alcohol, 1 with heroin, 1 with marijuana, and 3 with mixed substances). 9 adults were men; age range was 22-42 years; 6 were African-American, 5 were white, and 2 were Hispanic; substance abuse range was from 6-27 years; facility residency was from 4-28 months; and 3 had graduated from the programme.

\section{Methods}

Participant observation and semistructured interviews lasted 1.5-2 hours. Audiotaped interviews were transcribed and analysed using grounded theory.

\section{Main findings}

Participants felt that recovering from substance abuse was similar to changing careers and involved 4 progressive stages: enter- ing the programme, learning the programme, working with the programme, and gaining control.

Entering the programme was either voluntary or court ordered and involved a decision to be part of the programme despite its reputation for being difficult and long. During previous treatment programmes, participants often "burned bridges" with family and care providers and therefore had little support.

Learning the programme involved a 3 step process of resisting, going along, and letting go of self. Initially participants were pessimistic but curious to learn the programme's rules, tools, attitudes, behaviour, and skills needed for right living. This took time and dedicated study.

Working with the programme was seen to be hard work and involved 4 steps: confronting self, remembering the former life, learning how to communicate, and keeping busy. Participants had increased responsibility and employment inside or outside the facility.

Gaining control was the re-entry phase and occurred 18 months after entry. The goal was to contemplate and plan a life after the programme. The 5 stages were extracting a self, redirecting the self, remaining vigilant, giving back, and planning a life. Assessing and developing the good within was an important part of this phase. Short and long term goals were developed and re-entry was anticipated and viewed as living a "normal life."

\section{Conclusion}

Recovering from substance abuse in a therapeutic community was analogous to changing careers, with incremental stages in the redirection of self to a more productive lifestyle.

Source of funding: University of Texas Health Sciences Center at Houston, School of Nursing.

For correspondence: $\operatorname{Dr} M$ T Marcus, Department of Nursing Systems and Technology, School of Nursing, University of Texas Health Science Center at Houston, 1100 Holcombe, Suite 5.516 HMB, Houston, TX 77030, USA. Fax +1 7135002142

\section{Commentary}

The grounded theory research study by Marcus of the subjective experience of recovery from substance abuse within a therapeutic community makes an illuminating contribution to the complex phenomenon of recovery. She provides a thorough description of the developmental change process in recovery and also a view of how the process of living in a therapeutic community affects change. It adds substantially to the more objective data that previous studies have provided in trying to understand this major threat to public health. Studies of subjective experiences, once less valued, provide nurses with fertile ground to enhance deeper understanding and to develop and direct appropriate interventions.

Therapeutic communities were used in Britain after the second world war to treat many veterans who needed psychiatric care when few trained professionals were available. Positive outcomes were reported but little has been written about the use of therapeutic communities.

As nursing moves away from the focus on pathology towards a concentration on drawing forth patients' strengths and resources, new possibilities of interaction and relationship become possible. The study identifies that, unlike other therapeutic communities where character change is the goal, residual strengths of the previous self were valued to achieve success. Just as with individuals who are recovering from chronic and persistent mental illness, there is a need to identify in the experience of self as a person-the profound experience of having an illness that has consumed the self. Nursing inter- ventions might be targeted to the specific stage of recovery and could acknowledge the individual's strengths and differences.

Using the metaphor of changing careers may have some appeal for those who work with other populations such as cardiac patients who are struggling with lifestyle changes. Although access to long term, financially accessible therapeutic community settings may be limited in many regions, other groups of individuals with such health problems as obesity, alcohol misuse, and acquired physical disabilities might be studied to test furtherand expand the aptly named career change phenomenon.

Donna Tweedell, RN, MSN Clinical Nurse Specialist Hamilton Psychiatric Hospital Hamilton, Ontario, Canada 